\title{
Development of an Interspecific Hybrid (Triploid) by Crossing Gossypium hirsutum and G. arboreum
}

\author{
Muhammad Shafique Tahir ${ }^{1 *}$, Noor-UI-Islam Khan ${ }^{1}$ and \\ Sajid-Ur-Rehman ${ }^{2}$ \\ ${ }^{1}$ Cotton Research Institute, AARI, Jhang Road, Faisalabad, Pakistan \\ ${ }^{2}$ Agricultural Biotechnology Research Institute, AARI, Faisalabad, Pakistan
}

Received April 12, 2010; accepted March 28, 2011

\begin{abstract}
Summary The 2 species G. hirsutum and G. arboreum were crossed and triploid hybrid (AAD) was developed as a first step of crossing. The triploid is sterile and its hybridity was confirmed with morphological traits and DNA based SSR markers. The chromosome analysis at metaphase-I exhibited 13AA bivalents and 13D univalents at most of the PMC's studied which also confirmed its triploid nature. The mean chromosome pairing association was recorded as $12.90 \%$ (univalent), $10.45 \%$ (bivalent), $0.45 \%$ (trivalent), and $0.48 \%$ (tetravalent). The formation of multivalents depicted genome association between 2 species. The further utility of synthesized triploid with respect to the development of $\mathrm{CLCuV}$ resistant introgression genotypes is discussed
\end{abstract}

Key words Triploid, G. hirsutum, G. arboreum, Interspecific hybrid, Cotton leaf curl virus (CLCuV), In vitro.

Cotton is believed to be life line of the national economy of the country. During the last crop season, cotton staked in agriculture with $7.4 \%$ and $22.7 \%$ in value addition of major crops, grew modestly by $1.4 \%$ and its production, at 11.8 million bales, is below par (GOP, 2008-09). Cotton cultivation is mainly confined to Punjab and Sindh provinces with a few pockets in Baluchistan in relatively hot and dry environments. Cotton production in the Punjab province of Pakistan is mainly under threat due to attack of insect pests and cotton leaf curl virus disease. Among the insects, sucking pests viz whitefly (Bamisia tabicii), jassids (Amrasca bigutulla) and thrips (Thrips tabaci Lind) are posing the most serious threats and their population has tremendously increased in the last 4 to 5 years. It has been estimated that sucking pests alone are responsible for a $25-30 \%$ loss in lint and seed yield (Parnell et al. 1949). The cotton leaf curl virus disease is also playing havoc in the cotton belt of Punjab and its incidence is endemic in Sindh province also.

All commercial varieties having earlier resistant genes CP15/2, LRA 5166, Cedix and Kivi1021 (Ul-Islam and Iqbal 2002) are in the grip of the cotton leaf curl virus, mainly due to a narrow genetic base of genotypes which are succulent and hence attract the sucking pests and bring disease into effect through its vector whitefly. Therefore, resistance/tolerance to cotton leaf curl virus is mandatory nowadays in every cotton breeding program. Concerted efforts are being made to defy virus attacks by screening the subsequent generations of G. hirsutum crosses at hotspots of disease and eventually selecting high yielding disease tolerant genotypes. The success to have outstanding tolerance against disease is very low. The difficulty in generating a resistant genotype is posed by the mutant versions and new recombinants of CLCV virus (Mansoor et al. 2003). Hence, strategies to harbor new disease resistant genes from diploid wild species of cotton (G. arboreum, $G$. anamolum) to cultivated upland cotton ( $G$. hirsutum) are being launched to develop cultivars that possess built-in resistance to sucking insects and cotton leaf curl virus. The diploid species $G$. arboreum, G. herbacium and G. anamolum are completely immune to cotton leaf curl virus.

\footnotetext{
*Corresponding author, e-mail: mstahir63@yahoo.com
} 
Therefore, the introgression of useful characteristics should be an important method (Liang et al. 2002, Saravanan et al. 2007). However, transfer of cotton leaf curl virus resistance from $G$. arboreum to $G$. hirsutum is a tedious process because of cross incompatibility, hybrid breakdown and hybrid sterility related to pliody differences. Earlier studies revealed that introgression improves the productivity, fibre properties (Tayyab 1990, Liang et al. 2002, Mergeai 2003) and resistance to pests and diseases (Lukefahr et al. 1971, Avila et al. 2005). The present investigation reports a triploid with genetic constitution. (AAD) as a first step in successful hybridization between $G$. arboreum and G. hirsutum. The new hybrid is totally self-sterile and its interspecific status was confirmed using SSR markers and chromosome analysis. Among the available DNA fingerprinting methods SSR technique is most reliable and easy to perform. The scientists have consensus that SSR is the best technique for hybrid identification in cotton (Asif et al. 2009) and rice (Ye-Yun et al. 2005).

\section{Materials and methods}

\section{Plant material}

An accession G. hirsutum C.V. S.A-6654 and G.arboreum C.V. FDH-228 were selected for interspecific hybridization from gene pool of Cotton Research Institute, Faisalabad, Pakistan and grown in field during crop season 2007-08 with standard agronomic practices. The crossing was made effected by using following scheme.

1. Male Parent: (G. arboreum $2 n .2 x=26)$

2. Female Parent: (G. hirsutum 2n. $2 x=52)$

\section{Crossing procedure and ovule culture}

The appropriate flower buds were hand emasculated during late evening and pollinated in the next morning. The mixture of growth regulators at the concentration of $50 \mathrm{mg} 1^{-1} \mathrm{NAA}$ and $100 \mathrm{mg}$ $1^{-1}$ GA were applied after pollination at the base of pedicle for 20 days to reduce boll shedding and have ovules with good developed endosperms. The bolls were collected, surface sterilized and ovules were cultured in vitro on modified MS medium as described by (Stewart and Hsu 1977, 1978). The seedlings derived from cultured embryos were raised in small pots containing compost under controlled conditions of the lab facility. After proper temperature conditioning, seedlings were transferred in earthen pots at greenhouses for their growth and development in a natural environment.

\section{Morphological characters}

Data was collected on morphological characters viz growth habit, stem color, leaf color, leaf incision, leaf shape, leaf texture, leaf veins, petiol color, leaf hairiness, leaf nectriness, stem pubescence, position of stigma, corolla color, anther color, stigma length, petal spot, petal length, petal width, bracket length, bracket width and anther number of parents and a triploid hybrid and compared to describe the hybridity of triploid.

\section{DNA based confirmation of hybridity}

Young leaves from a triploid and parents were collected early in the morning. The leaves were transported to the laboratory in ice and stored at $-20^{\circ} \mathrm{C}$. The standard CTAB method was used for DNA extraction (Doyle and Doyle 1990).

DNA concentration in the samples was measured using Nanodrop Spectrophotometer (ND1000 , Nanodrop, USA) at wave lengths of 260 and $280 \mathrm{~nm}$. Quality of DNA was observed by running 50 ng DNA on $0.8 \%$ agarose gel. The quantity of DNA was also confirmed by comparing with Quantification Standards Phage $\lambda$ DNA (Fermentas) on $0.8 \%$ agarose gel. All DNA samples 
were diluted to a working concentration of 15 $\mathrm{ng} / \mu \mathrm{l}$ and stored at $-20^{\circ} \mathrm{C}$ in $50 \mu \mathrm{l}$ aliquots. The heterozygosity of triploid was determined by using SSR primers from BNL series (http://www.cottonmarker.org/projects/bnl/ index.shtml). After literature search, 10 primers reported to be polymorphic in previous studies were selected for hybridity testing in the present study. Two SSR primers (Table 1) showed polymorphism and co-dominance clearly differentiating the parents and hybrid. The SSR primers were amplified in Eppendorf thermocycler (Mastercycler gradient, 96 wells) by following protocol used by Ullah (2009). The amplicons were resolved through electrophoresis using $3 \%$ high resolution agarose gel for $1 \mathrm{~h} 20 \mathrm{~min}$ at $100 \mathrm{~V}$. The amplicons were visualized under UV after staining with ethidium bromide using G Box, Syngene Gel documentation system. The first and eighth wells of the gel were loaded with 50 bp DNA ladder (Gene Ruler ${ }^{\mathrm{TM}}$, \#SM0373, Fermentas, USA) for determination of exact size of amplicons. Second, third and fourth well of the gel were $G$. arboreum, G. hirsutum and interspecific hybrid (triploid) respectively. The parents and triploid were repeated in the fifth, sixth and seventh wells of the gel with second primer.

\section{Chromosome studies}

The young flower buds of appropriate stages of both parents and a triploid were collected from a single plant between 7.30 to 8.30 a.m. and fixed in $95 \%$ ethanol : glacial acetic acid $(3: 1)$ for $48 \mathrm{~h}$ and then preserved in 70\% ethanol until use. Cytological smears of pollen mother cells (PMCs) were squashed in $1 \%$ acetocarmine on microscopic slides. After squashing of anthers, slides were slightly warmed to get good spread of chromosomes. The appropriate meiotic stages were photographed with Olympus research microscope.

\section{Results}

\section{Crossibility and ovule culture}

A few triploids have been obtained by first accomplishing hybridization and then culturing the ovules in vitro to maturity. The survival of triploid hybrids till maturity was poor which indicated the effect of zygotic barriers. The seed setting between $G$. hirsutum and G. arboreum crosses, ovules cultured and number of viable hybrids obtained are depicted in Table 2. Out of 210 cultured ovules only 5 could be established in the field.

\section{Morphological characters}

The triploid plant resembled the parental parent in habit. The expression of morphological characters of parents and triploid hybrid is depicted in Table 3. The growth habit, stem color, leaf incision, leaf shape, position of stigma, anther color and petal spot resembled the parental parent. However, corolla color expression was intermediate of both parents. The petal spot expression of parental parent was of light color and intensity in triploid (Fig. 1A-C). The expression of leaf shape in triploid hybrid is vigorous (Fig. 1D) The size of stigma height, petal length, petal width, bracket length and anther numbers in triploid were higher than both of parents (Table 3).

\section{DNA based confirmation of hybridity}

Out of 40 SSR primers used in the study, 4 SSR primers were found to be polymorphic between the 2 parents, and out of these, only 2 primers differentiated the hybrid from parents. The amplification profile of these primers showed that these are co-dominant. The results of extremely 
Table 2. Crossability, boll setting and embryo development in interspecific hybridization

\begin{tabular}{cccccc}
\hline Cross & $\begin{array}{c}\text { No. of flowers } \\
\text { crossed }\end{array}$ & $\begin{array}{c}\text { No. of bolls } \\
\text { with } \\
\text { large embryos }\end{array}$ & $\begin{array}{c}\text { No. of ovules } \\
\text { cultured }\end{array}$ & $\begin{array}{c}\text { No. of triploids } \\
\text { obtained }\end{array}$ & $\begin{array}{c}\text { No. of triploids } \\
\text { survived } \\
\text { till maturity }\end{array}$ \\
\hline G. hirsutum $\times$ G. arboreum & 80 & 39 & 210 & 12 & 5 \\
\hline
\end{tabular}

Table 3. Morphological characters of parents and hybrid (triploid)

\begin{tabular}{rllll}
\hline \hline Sr. No & \multicolumn{1}{c}{ Characters } & G. hirsutum & \multicolumn{1}{c}{ G. arboreum } & G. hirsutum $\times$ G. arboreum \\
\hline 1 & Habit & Annual & Annual/intermediate & Perennial/intermediate \\
2 & Stem color & Green & Light reddish & Light reddish \\
3 & Leaf color & Green & Green & Green \\
4 & Leaf incision & Shallow & Deep & Deep \\
5 & Leaf shape & Palmate & Okra & Okra \\
6 & Leaf texture & Medium smooth & Medium smooth & Medium smooth \\
7 & Leaf veins & Thick prominent & Thick prominent & Thick prominent \\
8 & Petiol color & Green & Green & Green \\
9 & Leaf hairiness & Slight hairy & Absent & Slight hairy \\
10 & Leaf nectarines & Present & Present & Present \\
11 & Stem pubescence & Slight hairy & Slight hairy & Slight hairy \\
12 & Position of stigma & Protruded & Embedded & Embedded \\
13 & Corola color & Cream & Yellow & Creamy with yellow shade at base \\
14 & Anther color & Cream & Yellow & Yellow \\
15 & Stigma height & $1.5 \mathrm{~cm}$ & $1.4 \mathrm{~cm}$ & $1.8 \mathrm{~cm}$ \\
16 & Petal spot & Absent & Dark red & Light pink \\
17 & Petal length & $2.7 \mathrm{~cm}$ & $2.5 \mathrm{~cm}$ & $3.8 \mathrm{~cm}$ \\
18 & Petal width & $3.1 \mathrm{~cm}$ & $2.4 \mathrm{~cm}$ & $4.1 \mathrm{~cm}$ \\
19 & Bracket length & $1.5 \mathrm{~cm}$ & $1.5 \mathrm{~cm}$ & $2.5 \mathrm{~cm}$ \\
20 & Bracket width & $1.0 \mathrm{~cm}$ & $1.6 \mathrm{~cm}$ & $1.0 \mathrm{~cm}$ \\
21 & Anther number & 58 & 65 & 75 \\
\hline
\end{tabular}

polymorphic SSR primers BNL-1317 and BNL-2634 are depicted in Fig. 1F. These results clearly showed that sample given in the fourth and seventh lane is hybrid of parents (G. arboreum and $G$. hirsutum). As with primer BNL-1317, first parent amplified DNA fragment of $185 \mathrm{bp}$ compared to the DNA fragment of $200 \mathrm{bp}$ which was amplified by the second. This primer amplified different size fragments in the parents from the same loci of the genome showing the presence of different alleles of the same gene in 2 parents. This was confirmed from the amplification profile of the hybrid with primer BNL-1317 which showed the presence of both alleles in the hybrid. This loci depicted co-dominance and hybridity status of the offspring was confirmed from these results. Both of these primers individually can be used in hybridity confirmation. The results of the present study not only confirmed the hybridity status but also proved that SSR technique may successfully be used in such cases.

\section{Cytogenetics studies}

The meiosis in both G. arboreum (AA) and G. hirsutum (AADD) was regular and normal and both species showed 13 and 26 bivalents at Metaphase- 1 respectively (data not presented here). Theoretically, 13AA bivalents and 13D univalents are expected at metaphase-I in triploid hybrid. Therefore, expected configuration (13II+13I) was maximum and observed in $34.48 \%$ of MPC's, thus confirmed triploid hybrid (Fig. 1E and Table 4). Univallent, bivallent, trivalent and quardvallent chromosome associations were also recorded at various frequencies among 29 PMC's as depicted in Table 4. In the triploid under study, the number of univalents and bivalents ranged 


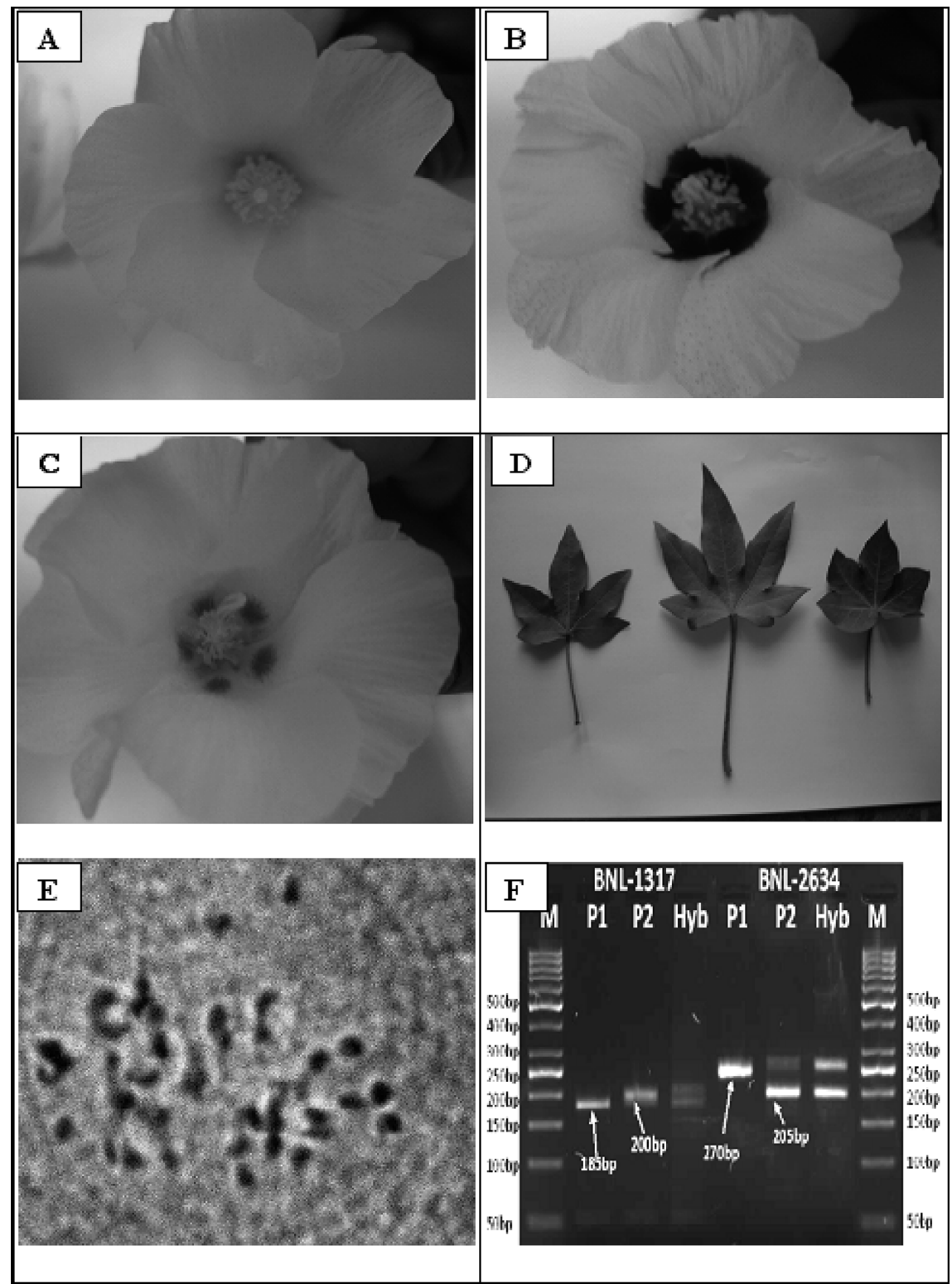

Fig. 1. Interspecific hybridization between $G$. hirsutum and G. arboreum

A) Flower of G. hirsutum, B) flower of G. arboreum, C) flower of triploid hybrid, D) left to right leaves of G. arboreum, triploid hybrid and G. hirsutum, E) 39 chromosomes (26A, 13D) in triploid hybrid. F) gel picture showing DNA amplification profile of parents and hybrid with polymorphic SSR primers BNL-1317 and BNL-2634 (Left to Right) M-50bp DNA ladder, P1-G. arboreum, P2G. hirsutum, Hyb-triploid hybrid)

from 12 to 14 and 8 to 13 with an average of 12.90 and 10.45 respectively. The number of trivalents and quardvallents ranged from 0 to 1 with an average of 0.45 and 0.48 respectively.

\section{Discussion}

The present investigation was primarily carried out to synthesize a triploid (AAD) as a first step of hybridization between 2 species $(G$. hirsutum $\times G$. arboreum). We report success in the 
Table 4. Cytological observation of chromosome association in triploid hybrid of G. hirsutm $\times$ G. arboreum

\begin{tabular}{cccccc}
\hline \hline \multicolumn{5}{c}{ Type and No. of associations per PMCs } \\
\hline PMC studied & I (Univalent) & II (Bivalent) & III (Trivalent) & IV (Tetravalent) & \% PMCS \\
\hline 10 & 13 & 13 & - & - & 34.48 \\
8 & 12 & 10 & 1 & 1 & 27.58 \\
6 & 13 & 8 & 1 & - & 20.68 \\
5 & 14 & 9 & 13 & 0.48 & - \\
Total=29 & 374 & 10.45 & 0.45 & - \\
Mean & 12.90 & & & 14 & - \\
\hline
\end{tabular}

development of such a triploid hybrid. This triploid is sterile due to ploidy barriers and variation of the chromosomes. Upland cotton (Gossypium hirsutum L.) is reproductively isolated from $G$. arboreum L. via post-zygotic breeding barriers (Sacks 2008). Therefore, in further studies, polyploidisation of triploid (AAD) will be carried out for its fertility restoration (Stewart 1995). The normal hybridization between diploid and tetraploid species is hindered due to differences in ploidy level and biological barriers, therefore, photohormones viz GA3 and NAA were sprayed in combination after pollination to enhance fertilization and metabolism in generative organs of maternal plant (Liang et al. 2002). The results showed that number of ovules with good developmental endosperm were cultured to maturity. The fertilized ovules were of bigger size and composition of medium described by Stewart and Hsu (1977) for seedling development worked perfectly and permitted embryos for active root and shoot formation. Our investigations demonstrated that $5.7 \%$ of cultured ovules developed into seedlings. The ovules with degenerated and less vigorous embryos did not germinate. The haploid nature of triploid was established with morphological, cytological and DNA based data. The synthesized triploid is intermediate in nature. The partial dominance type of expression of corolla color and petal spot make it distinct from its parents, which is in accordance with the observations of Sacks (2008). Moreover, stem color, leaf incision, leaf shape, position of stigma, anthor color, of $G$. arboreum were dominant as the F1 triploid (AAD) exhibited these characters. The transgressive effect for stigma height, petal length, petal width, bracket length and anther numbers is also observed in triploid. The parents and triploid were also subjected to cotton leaf curl virus stress by growing them near hotspots of the disease. Since whitefly is a vector for disease, therefore its infestation was not controlled chemically during crop season. The $G$. hirsutum completely succumbed to disease and absolutely no symptoms of $\mathrm{CLCuV}$ appeared on $G$. arboreum and triplod. From this, it is assumed that CLCuV resistance of $G$. arboreum can be transferred to G. hirsutum by carefully developing disease resistant introgression lines. The strategy will be based on the restoration of the fertility status of the triploid by chromosome doubling and repeated backcrossing of hexaploid with disease susceptible, quality fibre and high yielding $G$. hirsutum cultivar. The cytogenetic determination of subsequent tetraploid segregants and DNA based marker assisted selection of $\mathrm{CLCuV}$ resistant genes would be beneficial in the development of disease resistant introgressed genotypes. The DNA based data is also reliable for identification of interspecific hybrids because the hybrids between tetraploid and diploid is distinct from its parents for chromosome number and pairing. Utilized SSR markers support the hybridity of triploid, which is in accordance with earlier reports of Mehetre et al. (2004) and Saravanan (2007). The inheritance of SSR markers of both parents was depicted in hybrids which explains the presence of parts of parental genomes in the triploid hence confirming the success of hybridization among 2 species.

The cytogenetics analysis depicted 39 chromosomes (26A, 13D) in triploid. The main purpose of cytogenetic analysis was to confirm the hybrid nature of triploid which is fairly achieved in the present investigation. Some of the PMCs were further studied to have a look on pairing behavior of 
chromosomes in triploid with genetic constitution AAD. The mean pairing association $12.90 \%$ (univalent), $10.45 \%$ (bivalent), $0.45 \%$ (trivalent), and $0.48 \%$ (tetravalent) was depicted in our data which did not differ much from earlier report of Vafaie-Tabar and Chandarashekaram (2007). The presence of trivalents and quardvalents indicated pairing affinity exists between genomes involved. Since, both genomes (A and D) of triploid posses different number and size, therefore, homeolgous chromosomes rarely pair due to different size. The 13 chromosome observed in this study belongs to $\mathrm{D}$ genome and their univalents behavior can be attributed due to asynapsis or desynapsis. The difference in degree of chromosome condensation can be the main force controlling regular pairing behavior in Gossypium (Endrizzi 1962).

\section{References}

Avila, C., Stewart, J. and Robbins, R. T. C. 2005. Reniform nematode resistance in Asiatic Cotton Breeding Cotton Incorporated Breeding Cotton for Resistance to Nematodes Presentations on website: http://www.cottoninc.com/ 2005BreedingCottonResistanceNematodesProceedings/

Asif, M., Rahman, M., Mirza, J. I. and Zafar, Y. 2009. Parentage confirmation of cotton hybrids using molecular markers. Pak J Bot, 41: 695-701.

Doyle, J. J. and Doyle, J. L. 1990. Isolation of plant DNA from fresh tissue. Focus 12: 13-15.

Endrizzi, J. E. 1962. The diploid like cytological behavior of tetraploid cotton. Evolution 16: 325-329.

GOP, 2008-09. Economic survey of Pakistan: Finance Division, Government of Pakistan, pp. 19.

Liang, Z., Zhong, W., Qou, Z., Zhao G., Wang J. and Wang, L 2002. Creation of the technique of interspecific hybridization for breeding in cotton. Sci. China, C, Life Sci. 45: 331-336.

Lukefahr, M. J., Houghtaling, J. E. and Graham, H. M. 1971. Suppression of heliothis population glabrous cotton strains. J. Econ. Entomol. 64: 486-488.

Mansoor, S., Briddon, R. W., Bull, E. S., Bedford, I. D., Bashir, A., Hussain, M., Saeed, M., Zafar, Y., Malik, K.A., Fauquet, C. and Markham, P. G. 2003. Cotton leaf curl disease is associated with multiple monopartite begomoviruses supported by single DNA $\beta$. Arch. Virol. XX: 1-18.

Mehetre, S. S., Gomes, M., and Eapen, S. 2004. RAPD analysis of hybrid nature of the offspring of Gossypium hirsutum $\times$ G. raimondii. Curr. Sci. 87: 25-28.

Mergeai, M. 2003. Forty years of genetic improvement of cotton through interspecific hybridization at Gembloux Agricultural University: Achievement and prospects. In Proceedings of the World Cotton Research Conference-3, March 9-10, CAPE, Town. RSA. pp. 119-133.

Parnell, F. R., King, H. E. and Ruston, D. F. 1949. Jassid resistance and hairiness of cotton plant. Bull. ent. Res. 39: $539-575$.

Sacks, E. J. 2008. Ovule Rescue Efficiency of Gossypium hirsutum $\times$ G. arboreum Progeny from Field-Grown Fruit is Affected by Media Composition and Antimicrobial Compounds. Plant Cell Tissue Organ Cult. 93: 15-20.

Saravanan, N. A., Ram, S. G., Thiruvengadam, V., Ravikesavan, R. and Raveendran, S. 2007. Production and fertility restoration of an interspecific hybrid between Gossypium hirsutum L. and G. raimondii U. Cytologia 72: 195-203.

Stewart, J. M. and Hsu, C. L. 1977. In-ovule embryo culture and seedling development of cotton (Gossypium hirsutum L.) Planta 137: 113-117.

— and - 1978. Hybridization of diploid and tetraploid cottons (Gossypium sp.) through in-ovule embryo culture. J. Hered. 69: 404-408.

Stewart, J. M. C. D. 1995. Potential for crop improvement with exotic germplasm and genetic engineering. In: Challenging the future. Constable G. A. and N. W. Forrester (eds), Proceeding of the world cotton research conference-I, Brishbane, Australia, February 14-17, Melbourne. pp. 313-327.

Tayyab M. A. 1990. Use of wild species in heterosis breeding of cotton. PKV Res. J. 14: 1-4.

Ul-Islam, N. and Iqbal, M. Z. 2002. Future breeding goals and strategies to improve cotton in Pakistan. The Pakistan Cottons 46: 43-61.

Ullah, I. 2009. "Molecular Genetic studies for Drought tolerance in cotton" Ph.D thesis Quaid-i-Azam University, Islamabad.

Vafaie-Tabar, M. and Chandrashekran, S. 2007. Meiosis in a triploid hybrid of Gossupium: high frequency of secondary bipolar spindles at metaphase II. J. Genet. 86: 45-49.

Ye-Yun, X., Z. Zhan, X. Yi-ping and Y. Long-ping. 2005. Identification and purity test of super hybrid rice with SSR molecular markers. Zhongguo Shuidao Kexue. 12: 7-12. 